\title{
Gaussian process regression prediction-based dynamic risk negotiation strategy
}

\author{
Jun $\mathrm{Hu}^{1,2}$ \\ ${ }^{1}$ College of Information Science and Engineering \\ Hunan University, Changsha, China \\ 2 \\ Guangxi Key Laboratory of Trusted Software, \\ Guilin University of Electronic Technology, \\ Guilin, China \\ hujun_111@hnu.edu.com
}

\author{
Li Zou $^{1}$ \\ ${ }^{1}$ College of Information Science and Engineering \\ Hunan University \\ Changsha, China \\ Zouli19881229@163.com
}

\begin{abstract}
In this paper, we use Gaussian process regression to predict the opponent concessions, and introduce the dynamic risk mechanism in Agent negotiation. We can change the risk factor by the utility of opponents and set a threshold in this dynamic risk mechanism. Dynamic risk strategies associated with opponent concessions prediction can not only dynamically change the risk attitude according to the utility of opponents, but also obtain a higher utility value in the negotiation. We establish this negotiation model and run it in the Generic Environment for Negotiation with Intelligent multi-purpose Usage Simulation (GENIUS), and the results prove the performance of our Agent is superior to other Agents.
\end{abstract}

Key words- multi-issue negotiation; Gaussian process regression; dynamic risk strategy; concession strategy

\section{INTRODUCTION}

Negotiation is a process of agreement through concession on certain issues between Agents [1]. We can see that there are conflicts in the process of negotiation. In such seemingly contradictory process of negotiation, concession is the most critical factor. Agents involved in negotiation are looking for a mutually acceptable proposal, which can be allowed by the other side Agent. In recent years, negotiation is central issue in many fields, like artificial intelligence, mathematics, and economics and so on. In the field of artificial intelligence, researchers study aspects including negotiation strategies, negotiation model and Multi-Agent systems. The purpose of this paper is to study the negotiation strategy and model under the setting of bilateral multi-issue. Automated Negotiating Agent Competition (ANAC) provides us with the method of designing Agent and the bilateral multi-issue negotiation environment. In the Generic Environment for Negotiation with Intelligent multi-purpose Usage Simulation (GENIUS) [2], we designed a special Agent called RiskAgent, which predicts the opponent's concessions based on Gaussian process and introduces dynamic risk mechanism. On the basis of the previous, we take that Agent in the setting of bilateral multi- issue negotiation and negotiation tournament to negotiate with other Agents who participated in ANAC.

\section{RELATED RESEARCH}

Researchers participated in ANAC competition designed many outstanding Agent that using different negotiation strategy. JC Harsanyi et al [3] proposed a generalized Nash solution to bilateral negotiation in complex environment, but this method did not take into account the constraint of negotiation time. S. Kawaguchi et al [4] considered a negotiation strategies based on rival historical information to determine their own concession strategies and proposed utilities. Mai Ben Adar et al [5] proposed a negotiation strategy based on meta-learning. Liviu Dan Serban et al [6] designed the AgentFSEGA is a time constraint reasoning model. Bo An et al [7] designed a Agent model Yushu, based on heuristics. Characteristics of the model is choose the smallest fully concession strategy in the negotiation. Katsuhide Fujita et al [8] designed Nozomi negotiation model which is able to keep track of the opponent's concession, but cannot predict the opponent utility function. Colin et al [9] proposed a negotiation strategy based on Gaussian regression analysis to predict the opponent's compromise, which is mainly analyzing the proposal utility of opponents. This method does not consider the risk factors associated with the proposed utility of opponents, when setting rates concession. Negotiation model presented in this paper based on the Colin's model, in order to improves the efficiency of Agent negotiated by adding dynamic risk.

\section{NEGOTIATION STRATEGY}

\section{A. Threshold-based dynamic risk mechanism}

Generally believed, the attitude of Agent towards risk can be divided into three categories: averse, seeking and neutral [10]. When the risk function is a power function expressed as $R(u)=u^{r}$, if $0<r<1$, we call it risk-averse; if $r=1$, it is risk-neutral; if $r>1$, it is risk-seeking. According to this characteristic of the risk function, we applied it to a real-time negotiation and associated with the utility of opponents to determine our risk strategy so as to

Supported by Guangxi Key Laboratory of Trusted Software (No: kx201333). 
achieve better negotiation results. The value of risk factor $r$ determines the attitude of risk function.

In order to associate the utility function with the risk function to achieve dynamic risk mechanism, we do not set the value of $\mathrm{r}$, but set $r=1+u_{\text {diff }}$ to achieve dynamic risk mechanism. Wherein, $u_{\text {diff }}$ is the difference between the utility of opponents' offer and the value of threshold $u_{e}$, where $u_{\text {diff }}$ is expressed as

$$
u_{\text {diff }}=u-u_{e}
$$

We set the value of $u_{e}$ to decide the basic risk attitude of our Agent. We set the utility of an offer as $\mathrm{u}$, if $u<u_{e}$, then $u_{\text {diff }}<1$, so the Agent is risk-averse. Risk-neutral and risk-seeking are determined by the same way. Therefore, our dynamic risk function is expressed as

$$
R_{\text {dynamic }}(u) \begin{cases}\text { risk-averse }, & u<u_{e} \\ \text { risk-neutral, } & u=u_{e} \\ \text { risk-seeking, } & u>u_{e}\end{cases}
$$

Which, risk-averse means the risk attitude of Agent is averse, risk-neutral means the risk attitude of Agent is neutral, risk-seeking means the Agent is eager to find risk. Thus, we risk attitude of our Agent depends on the opponent's utility value, if the utility value is very low, even lower than our threshold, then our Agent believes the utility of the offer is too low, then our Agent will reject the offer and make a counter-offer, and we consider the utility offered by the opponent has a high risk. Then our Agent will be able to get a higher utility value. Contrary, we think the utility offered by the opponent has a low risk.

\section{$B$. predict the opponent concessions}

According to the known information, our Agent predicts the opponent concessions through Gaussian process regression [11] and the risk attitude. For each offer, we want to record relevant information, including the time and the utility value, the utility value is calculated by our utility function. We get the prediction and its confidence by Gaussian process regression. The reason why we chose Gaussian process regression to predict is because its nature is completely determined by the mean and covariance functions. We use a linear mean function and Matérn covariance functions to complete the Gaussian process regression analysis $[9,11]$. Gaussian process output is a Gaussian distribution

$$
f\left(u ; \mu_{t}, \sigma_{t}\right)=\frac{1}{\sigma_{t} \sqrt{2 \pi}} e^{\frac{-\left(u-\mu_{t}\right)^{2}}{2 \sigma_{t}^{2}}}
$$

According to the characteristics of the Gaussian distribution, the mean $\mu_{t}$ indicates the most likely value of $u$ in time $t$, and the standard deviation $\sigma_{t}$ represents the prediction confidence of $\mu_{t}$. The information we need to know is deadline and discount factor. For every opponent offer $O_{o p p}$, we firstly recorded time and the utility value of the offer, and then we determine our risk factor $r$ based on the opponent utility values. We firstly obtained the mean $\mu$ and the variance $\sigma$ by Gaussian process regression, in order to predict the opponent concession strategy. According to the predicted value previously obtained and the risk factor, we can get the best time to give the offer and the corresponding utility value. According to $\mu_{t}$ and $\sigma_{t}$, we can calculate the best time $t_{\text {best }}=\arg \max _{t \in\left[t_{c}, t d l\right]} E(t)$ and the corresponding utility $u_{\text {tbest }}$. Where, $t_{c}$ is the current time, Agent's expected utility is $E(t)=\int_{0}^{1} D\left(f\left(u ; \mu_{t}, \sigma_{t}\right), t\right) d u$. Where, $f(\cdot)$ is the possible distribution of Gaussian process output $u$.Therefore, the utility which should be offered at time $t_{\text {best }}$ is given by

$$
u_{\text {best }}=\arg \max _{u \in[0,1]} E_{o f f e r}\left(u, t^{*}\right)
$$

Wherein,

$E_{\text {offer }}\left(u, t_{\text {best }}\right)=D\left(R_{\text {dynamic }}(u) F\left(u ; \mu_{t_{\text {best }}}, \sigma_{t_{\text {best }}}\right), t_{\text {best }}\right)$ is the expected utility, which determined by both the risk function and the cumulative distribution function of Gaussian regression.

\section{Selecting an offer with nonlinear calculation}

We need to choose a utility value to offer at the current time $t_{c}$. We will not choose $U_{\text {tbest }}$ as the utility of the offer, because it is too extreme by this way. We simply to concede nonlinearly between $\left(t_{l r}, U_{l r}\right)$ and $\left(t_{\text {best }}, U_{\text {tbest }}\right)$, where $t_{l r}$ is the time at which the regression was last performed and $U_{l r}$ is the target utility at that time. The target utility $U_{t}$ is given by

$$
U_{t}=U_{l r}+\left(t c^{2}-t l r^{2}\right) \frac{U_{t b e s t}-U_{l r}}{t_{\text {best }}{ }^{2}-t_{l r}{ }^{2}}
$$

The comparison of using nonlinear way to select the offer with using linear way is shown in Figure 1.

As can be seen from the figure, the nonlinear selection is to let this two point $\left(t_{l r}, U_{l r}\right)$ and $\left(t_{\text {best }}, U_{\text {tbest }}\right)$ in a quadratic function. Compared with the linear way, the advantage is that Agent can change the slope by $t_{c}$. When closer $t_{\text {best }}$ change sooner. When we get $U_{t}$, we want to ensure that as close as possible $U_{t}$. We first set $\Delta=0$, if $\left|U_{\text {offer }}-U t\right| \leq \Delta$, represents $U_{\text {offer }}$ within the desired range, otherwise the value of $\Delta$ is increased until the value 
$U_{\text {offer }}$ satisfies the condition. Then our Agent generates an offer.

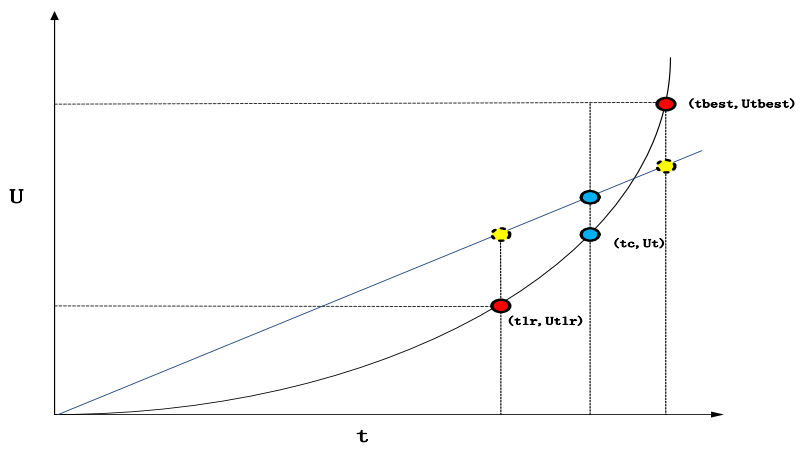

Figure 1. Comparison nonlinear with linear selection

\section{EXPERIMENTS DESIGN AND ANALYSIS}

In GENIUS platform, you can simulate the bilateral multi-issue negotiation as well as organize Agents to take turn to bilateral multi-issue negotiation. GENIUS platform is able to draw each utility values from each Agent negotiation, the results of the negotiation can be integrated to reflect the merits of the Agent.

First, we will use both former strategy (IAMhaggler) and dynamic risk strategy (RiskAgent) in travel (Chox vs Panny) domain to simulate a bilateral multi-issue negotiation. RiskAgent and IAMhaggler respectively as Agent A (chox) and Agent B (panny) to negotiate on Atmosphere, Amusement, Culinary, Shopping, Culture, Sport and Environment seven topics. We first set IAMhaggler as Agent A and set RiskAgent as Agent B, Figure 2 shows the negotiation results

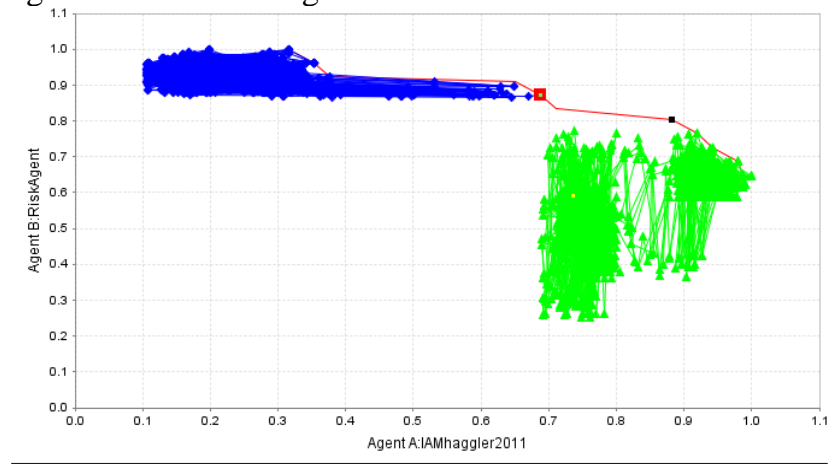

= all possible bids - Pareto efficient frontier $₫$ Agent A's bids $\rightarrow$ Agent B's bids $\boldsymbol{\|}$ Nash Point $\boldsymbol{\|}$ Kalai Point - Agreement = Last bid by $A$ = Last bid by $B$ - Agent A's reservation value - Agent B's reservation value

Figure 2. Negotiation results when RiskAgent is Agent B

In figure 2, the area covered by a blue diamond represents RiskAgent's offer records, the area covered by a green triangle indicates the offer record of IAMhaggler, and the red box represents agreement point. Figure 4.1 shows all offers are not exceeding the rightmost margin of Pareto, and reach an agreement on the Pareto optimal boundary. At the end of negotiations, IAMhaggler get utility value 0.687143 and RiskAgent get utility value 0.873176 . Similarly, we then respectively set Agent A as RiskAgent and Agent B as IAMhaggler2011. At the end of negotiations, IAMhaggler get utility value 0.802015 , RiskAgent get utility value 0.763615 . We compare the concessions situation between two Agents through the average utility values statistics of every 1000 times during the course of negotiation. Figure 3 shows the statistical results

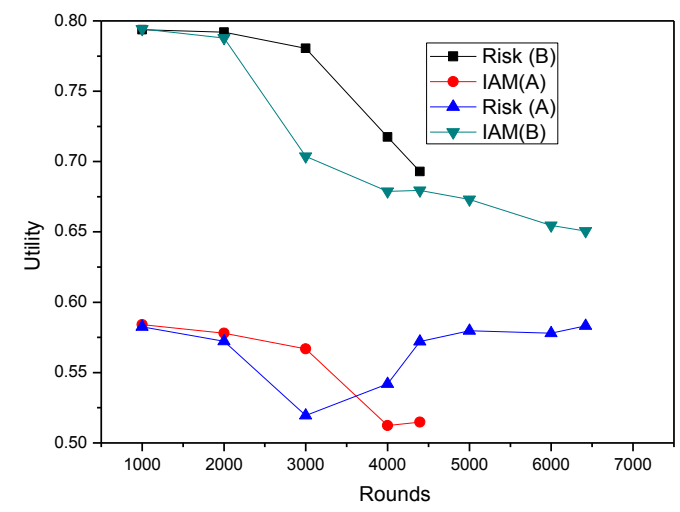

Figure 3. Bilateral multi-issue negotiation concessions statistics

Figure 3 shows when IAMhaggler2011 as Agent A, Agent sides have been made concessions until the end of negotiation and a relatively small number of interactions. When RiskAgent as Agent A, IAMhaggler still made concessions all the time but RiskAgent use aggressive tactics after round 3000, the value of RiskAgent's offers keeps a upward trend until the final agreement, which shows dynamic risk strategies can achieve higher utility value. In addition, RiskAgent set into both Agent A and Agent B obtained a higher utility value compared to IAMhaggler2011 under the same conditions. The results prove that the use of dynamic risk strategies is superior to fixed risk strategy in Agent negotiation.

In order to ensure the integrity and fairness of the experimental results, we run a tournament in GENIUS to test the performance of our Agent. We chose two negotiation domains England (Agent A) VS Zimbabwe (Agent B) and ITex (Agent A) VS Cypress (Agent B) to make experiment analysis. Six Agents negotiate in the tournament, which are AgentFSEGA, AgentK, Nozomi, Yushu, IAMhaggler and RiskAgent. In this competition, the utility of each Agent $U_{\varphi}(a)$ in the domain $\varphi$ is calculated according to the following formula

$$
U_{\varphi}(a)=\frac{\sum_{a^{\prime} \in A \cdot a^{\prime} \neq a} U_{\varphi}\left(a, a^{\prime}\right)}{|A|-1}
$$

Where $U_{\varphi}\left(a, a^{\prime}\right)$ represents the utility values of negotiations between Agent $a$ and Agent $a^{\prime}$ in domain $\varphi$, $A$ is the set of all Agents. 
By calculating the effectiveness of this method, we firstly make utility value statistics of all six Agents in domain England VS Zimbabwe. The negotiation results of Six Agents are shown in Table I

TABLE I. NEGOTIATION RESULTS COMPARISON IN D1

\begin{tabular}{|l|c|c|c|c|}
\hline Domian & Model & $\begin{array}{c}\text { Average } \\
\text { Utility }\end{array}$ & $\begin{array}{c}\text { Average } \\
\text { Time }\end{array}$ & $\begin{array}{c}\text { Average } \\
\text { Efficiency }\end{array}$ \\
\hline \multirow{3}{*}{$\begin{array}{l}\text { D1: } \\
\text { England }\end{array}$} & AgentFSEGA & 0.7459652 & 0.420556 & 1.773759602 \\
\cline { 2 - 5 } ZS & AgentK & 0.8248038 & 0.783328 & 1.05294817 \\
\cline { 2 - 5 } & Nozomi & 0.7758153 & 0.635121 & 1.221523684 \\
\cline { 2 - 5 } & Yushu & 0.751769 & 0.796332 & 0.944039642 \\
\cline { 2 - 5 } & IAMhaggler & 0.7350204 & 0.584579 & 1.257350055 \\
\cline { 2 - 5 } & RiskAgent & 0.8315214 & 0.644211 & 1.29075941 \\
\hline
\end{tabular}

As can be seen from the table, in this domain, the utility of RiskAgent is 0.8315214 , ranked first, the utility of AgentK is 0.8248038 , ranked second, and the utility Nozomi is 0.7758153 , ranked three. In ITex VS Cypress domain, the negotiation results of six Agents are shown in Table II

TABLE II. NEGOTIATION RESULTS COMPARISON IN D2

\begin{tabular}{|l|c|c|c|c|}
\hline Domian & Model & $\begin{array}{c}\text { Average } \\
\text { Utility }\end{array}$ & $\begin{array}{c}\text { Average } \\
\text { Time }\end{array}$ & $\begin{array}{c}\text { Average } \\
\text { Efficiency }\end{array}$ \\
\hline \multirow{3}{*}{$\begin{array}{l}\text { D2: } \\
\text { ITex }\end{array}$} & AgentFSEGA & 0.6263367 & 0.723566 & 0.865625 \\
\cline { 2 - 5 } Cypress & AgentK & 0.5165633 & 0.834928 & 0.618692 \\
\cline { 2 - 5 } & Nozomi & 0.7362431 & 0.854738 & 0.861367 \\
\cline { 2 - 5 } & Yushu & 0.6548945 & 0.890173 & 0.735694 \\
\cline { 2 - 5 } & IAMhaggler & 0.6827869 & 0.802154 & 0.851192 \\
\cline { 2 - 5 } & RiskAgent & 0.7420843 & 0.787693 & 0.942098 \\
\hline
\end{tabular}

Table II shows the top three Agents are RiskAgent (0.7420843), Nozomi (0.7362431) and IAMhaggler2011 (0.6827869). In this Domain, RiskAgent still get the highest utility.

Based on the above results of the tournament, the comparison of the utility in two domains is shown in Figure 4

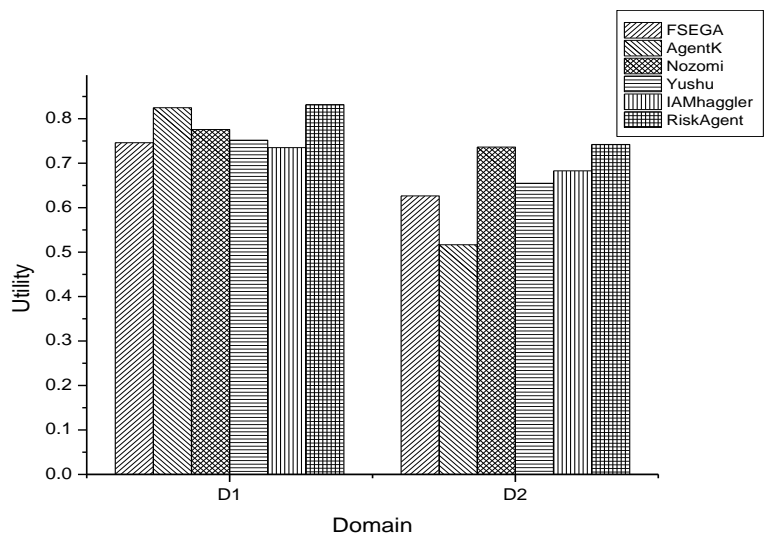

Figure 4. The comparison of all Agents' utility in two domains

The results of the experiments proves that the use of dynamic risk strategy have a clear advantage in the negotiation, because it can dynamically change the risk factor in accordance with the utility value offered by the opponent, and the use of non-linear calculations allows the utility closer to the expected utility. We change the risk strategy through analyzing our opponents' offers and concessions. So the performance has greatly improved compared to IAMhaggler2011, and significantly better than the time-based constraints Agent FSEGA and the Yushu which choose the smallest concession strategy. Meanwhile, the experimental results show that the use of dynamic risk strategy is superior to the use of other strategies.

\section{CONCLUSION}

In this paper, we proposed a negotiation strategy which introduces the threshold-based dynamic risk mechanism in the negotiation model proposed by Colin et al which is based on Gaussian regression to predict opponent's concessions. Because of the risk factors associated with the utility of the opponents, in the whole negotiation process Agent analyzes of the opponent concession strategy, and need to determine the concession rates and concessions strategy according to the opponent, so that the better Agent negotiation performance can be reached. Finally, we analysis the comparative empirical results, and verify the efficiency of the negotiation strategy.

\section{REFERENCES}

[1] N R Jennings, P Faratin, A R Lomuscio, et a1.Automated negotiation: Prospects, methods and challenges [J].International Journal of Group Decision and Negotiation, 2001.10(2):199-215

[2] K Hindriks, CM Jonker, S Kraus, R Lin, and D Tykhonov. GENIUS: negotiation environment for heterogeneous agents. Proc. of the Eighth Int.Joint Conf. on Autonomous Agents and Multiagent Systems, 2:1397-1398, 2009

[3] J.C. Harsanyi and R. Selten. A generalized Nash solution for twoperson bargaining games with incomplete information. Management Science, 18(5):80-106, January 1972

[4] Shogo Kawaguchi, Katsuhide Fujita, Takayuki Ito. AgentK: Compromising Strategy based on Estimated Maximum Utility for Automated Negotiating Agents. New Trends in Agent-Based Complex Automated Negotiations Studies in Computational Intelligence Volume 383, 2012,pp 137-144

[5] Mai Ben Adar,Nadav Sofy,Avshalom Elimelech, Gahboninho: Strategy for Balancing Pressure and Compromise in Automated Negotiation, Complex Automated Negotiations: Theories, Models, and Software Competitions Studies in Computational Intelligence Volume 435, 2013, pp 205-208

[6] Liviu Dan Şerban, Gheorghe Cosmin Silaghi, Cristian Marius Litan. AgentFSEGA: Time Constrained Reasoning Model for Bilateral Multi-Issue Negotiations. New Trends in Agent-Based Complex Automated Negotiations Studies in Computational Intelligence, Volume 383, 2012, pp 159-165

[7] Bo An, Victor Lesser, Yushu: A Heuristic-Based Agent for Automated Negotiating Competition. New Trends in Agent-Based Complex Automated Negotiations Studies in Computational Intelligence Volume 383, 2012, pp 145-149

[8] Kawaguchi S, Fujita K, Ito T. AgentK: Compromising Strategy Based on Estimated Maximum Utility for Automated Negotiating Agents[M]//New Trends in Agent-Based Complex Automated Negotiations. Springer Berlin Heidelberg, 2012: 137-144.

[9] C.R. Williams, V. Robu, E.H. Gerding, N.R. Jennings, Using Gaussian processes to optimize concession in complex negotiations against unknown opponents, in: Proc. of the Twenty-Second International Joint Conference on Artificial Intelligence, vol. 1, 2011, pp. $432-438$

[10] Lan Wu, Risk Theory, Peking University Press, Page 172-175.

[11] CE Rasmussen and CKI Williams. Gaussian Processes for Machine Learning. The MIT Press, 2006 